\title{
Efectividad de un programa de terapia de familia en niños asmáticos con familias disfuncionales
}

\author{
Dedsy Yajaira Berbesí Fernández ${ }^{\mathrm{a}}$, Margarita García Jaramillob, Ángela Segura Cardonac, \\ Ricardo Posada Saldarriaga ${ }^{d}$ y Paula Serna Escobare
}

\begin{abstract}
aDocente-investigadora. Grupo de Investigación Epidemiología y Estadística. Universidad CES. Medellín (Colombia)

'Docente-investigadora. Grupo de Investigación Observatorio de Salud Pública. Universidad CES. Medellín (Colombia) 'Docente. Grupo de Investigación Epidemiología y Estadística. Universidad CES. Medellín (Colombia) dJefe de Posgrados Clínicos. Grupo de Investigación Pediatría CES. Universidad CES. Medellín (Colombia) eEstudiante de Medicina. Universidad CES. Medellín (Colombia)
\end{abstract}

Correspondencia: Dedsy Yajaira Berbesí Fernández. Correo electrónico: dberbesi@ ces.edu.co

Recibido el 30 de agosto de 2013.

Aceptado para su publicación el 8 de octubre de 2013.

\section{RESUMEN}

Objetivo: Demostrar la efectividad de la terapia familiar como método adicional en el tratamiento del asma, para la mejoría de los síntomas, la función pulmonar y la disminución de las crisis en niños que forman parte de familias disfuncionales.

Diseño del estudio: Se realizó un estudio de intervención antes-después.

Emplazamiento: Seis instituciones de salud de la ciudad de Medellín, Colombia.

Participantes: Veinticinco niños con diagnóstico de asma y familias con disfunción familiar, en el año 2011.

Mediciones principales: Análisis antes y después de la intervención familiar utilizando las pruebas de Wilcoxon y McNemar. La significación estadística fue considerada con un valor de $\mathrm{p}<0,05$.

Resultados: Antes de la intervención el $68 \%$ de los niños tenían asma no controlada, y luego de la terapia familiar, al finalizar el año de seguimiento, la persistencia del asma no controlada fue del $4 \%$. Se reportó una gran mejoría de los síntomas clínicos tras la intervención en la familia, y la CVF y el VEF1 mostraron un aumento significativo $(p<0,01)$.

Conclusiones: La instauración de cambios en la dinámica familiar logra disminuir los síntomas, las crisis asmáticas y lleva a un mayor control de la enfermedad en los niños; es por esto que la evaluación de la dinámica familiar debe considerarse una parte fundamental en el abordaje integral del paciente pediátrico con asma.

PALABRAS CLAVE: Relaciones Familiares, Familia, Asma, Terapia Familiar.

\section{ABSTRACT}

Effectiveness of a family therapy programme in asthmatic children with dysfunctional families.

Objective: To show the effectiveness of family therapy as an additional method in the treatment of asthma, to improve the symptoms, lung function and a reduction of attacks, in children from dysfunctional families.

Design: A before-after intervention study was carried out.

Location: Six health institutions in the city of Medellín, Colombia.

Participants: Twenty-five children diagnosed with asthma and family dysfunction in 2011.

Main Surveyed Data: Analysis before and after family intervention using Wilcoxon and McNemar tests. The statistical significance was considered with a value of $p<0,05$.

Results: Prior to the intervention, $68 \%$ of the children were suffering from uncontrolled asthma and after family therapy, at the end of the follow-up year, uncontrolled asthma persistence was $4 \%$. A great improve in clinical symptoms was reported after family intervention, and FVC and FEV1 showed a significant increase $(p<0,01)$.

Conclusions: The establishment of changes in family dynamics achieves a reduction in symptoms and asthma attacks and brings a greater control of disease in children; for this reason, family dynamics assessment must be considered a fundamental part in the integral approach of paediatric asthma patients.

Key words: Family Relationships, Family, Asthma, Family Therapy. 


\section{INTRODUCCIÓN}

El asma es una de las enfermedades crónicas más comunes en el mundo. Se estima que 300 millones de personas se ven afectados por ésta. La prevalencia global del asma varía del $1 \%$ al $18 \%$ de la población en diferentes países, y se encuentra en aumento, especialmente en la infancia, con una edad de inicio alrededor de los dos años ${ }^{1}$. Se caracteriza por una inflamación y obstrucción crónica de la vía aérea, y síntomas como dificultad respiratoria, tos, sibilancias y opresión en el pecho. Su diagnóstico se realiza por medio de la historia clínica y la medición de la función pulmonar que permite la valoración de la severidad, generalmente utilizando la espirometría. Este evento es desencadenado por una hiperreactividad a factores como alérgenos, irritantes, agentes infecciosos, cambios ambientales, ejercicio, medicamentos y factores emocionales, estos últimos relacionados principalmente con la dinámica familiar ${ }^{1-3}$.

Desde el punto de vista fisiológico se cree que el nervio vago es el mediador de la reactividad de las vías respiratorias y las emociones. Las vías respiratorias superiores, inervadas por neuronas colinérgicas, pueden ser más sensibles a la emoción que las vías más pequeñas, por lo que diversas emociones y factores estresantes familiares pueden incrementar la resistencia respiratoria en el asma disparando mecanismos de broncoconstricción ${ }^{4}$.

La disfunción familiar es la incapacidad de la familia para enfrentar las crisis y cumplir algunas o todas sus funciones básicas (afecto, comunicación, adaptabilidad, resolución de problemas, participación y normas de convivencia) ${ }^{5,6}$. Se ha observado que factores familiares como el estilo de crianza y el funcionamiento de la familia están vinculados con los problemas de salud física como el asma en los niños ${ }^{7}$ al ser utilizados como un mecanismo de respuesta para la resolución de los problemas familiares ${ }^{5,6}$. Diversos autores han señalado el papel de la familia como modulador de la intensidad de los determinantes psicosociales que conducen a la instauración de una enfermedad asmática moderada y grave persistente en los niños, identificando la asociación entre estructura familiar y gravedad del asma infantil ${ }^{8-10}$.

Aunque el tratamiento del asma tiene un gran componente farmacológico por las causas biológicas de este evento, la terapia familiar es un coadyuvante que busca ofrecer a la familia recursos para adaptarse y hacer frente a los cambios en el sistema de la familia que acompaña a la aparición de la enfermedad ${ }^{11}$. Además de esto, su objetivo es identificar los factores familiares que podrían exacerbar los problemas de salud en los hijos, las relaciones entre sus integrantes y las dificultades entre ellos ${ }^{12}$ para ser intervenidos y producir cambios en la dinámica familiar y llevar a una mejoría de los síntomas de enfermedades psicosomáticas como el asma infantil.

En este estudio se busca demostrar la efectividad de la terapia familiar como método adicional en el tratamiento del asma para la mejoría de los síntomas, la función pulmonar y la disminución de las crisis en niños que forman parte de familias disfuncionales.

\section{SUJETOS Y MÉTODOS}

Se realizó un estudio de intervención antesdespués para analizar la presencia de crisis asmáticas después de una intervención familiar en niños asistentes a seis instituciones de salud de Medellín (Colombia) en el año 2011. Fueron seleccionados un total de 25 niños de entre 8 y 12 años con diagnóstico de asma y presencia de disfunción familiar.

En la primera fase se realizaron entrevistas al grupo familiar utilizando un formulario con preguntas demográficas, sociales y clínicas. Para determinar el diagnóstico de asma se utilizó los criterios de GINA, el examen de espirometría y la evaluación por pediatra neumólogo. Se tomaron como síntomas clínicos de enfermedad: tos, sibilancias, dificultad respiratoria, síntomas nocturnos y además la necesidad de utilización de $\beta 2$ agonistas. Se interrogó por la aparición de estos síntomas antes y después de la terapia familiar. La funcionalidad familiar se determinó con la escala de APGAR familiar, instrumento validado y específico ${ }^{13-15}$ y mediante entrevista con una médica especialista en terapia de familia. Todas las familias otorgaron autorización escrita mediante consentimiento informado y se verificó la capacidad de entendimiento, razonamiento y lógica del niño para responder a la entrevista por medio de la prueba RAVEN, realizada por una psicóloga.

El grupo seleccionado recibió el tratamiento habitual ordenado por la institución de salud. Adicionalmente las familias de los niños recibieron cuatro sesiones buscando el manejo y la adaptación a las crisis de los niños, mejorando la dinámica relacional de los miembros de la familia. La intervención familiar tenía varias estrategias: información médica, estrategia educativa, adaptación a la enfermedad e influencia de la dinámica familiar. Estos componentes se 
realizaron mediante la orientación y terapia familiar.

Inicialmente se creó un espacio con los niños y la familia para dar una información básica acerca de la enfermedad del paciente que incluyó definición de la enfermedad, diagnóstico, factores de riesgo, manejo de la enfermedad, complicaciones y la importancia de incluir a la familia en el manejo de la enfermedad.

El objetivo de la terapia familiar era lograr un cambio en las relaciones familiares disfuncionales del paciente asmático y en el curso y la evolución del asma para que éste no solo respondiera a la medicación sino que también se intervinieran los factores emocionales que empeoraban la enfermedad.

Dentro de la terapia familiar sistémica comenzamos a sustituir los modelos lineales por modelos de causalidad circular, es decir, no estudiando al individuo aislado sino buscando las conexiones entre el individuo y su familia. El modelo que se realizó durante las entrevistas clínicas fue el de la narrativa, donde el proceso terapéutico es visto como una conversación y las familias participan en la entrevista de una forma menos cohesiva ${ }^{16}$.

Para hacer este cambio de lo lineal a lo circular se realizan las siguientes etapas en la entrevista familiar basadas en las dimensiones que Slusky propone $^{17}$ : 1) conocimiento del paciente y la familia; 2) planteamiento y construcción del problema; 3 ) validación del síntoma o del problema por parte del paciente y su familia; 4) manifestación del tipo de ayuda y objetivos de la consulta; 5) establecer la dimensión del tiempo (explorar desde cuándo se presenta, bajo qué circunstancias, cuándo se agudiza); 6) establecer la dimensión del espacio no contextual lineal a lo contextual (qué pasaba en ese momento en la familia, en el colegio, con sus amigos); 7) determinar la dimensión de la causalidad (cambiar la historia centrada en las causas y quién tiene la culpa del problema o síntoma a los efectos de éste en el paciente y la familia); 8) determinar la dimensión de la interacción, es decir, de un contexto intrapersonal a uno interpersonal analizando efectos y consecuencias de estos en la historia del paciente (con qué relaciona lo que le pasa al paciente y a la familia con el síntoma); 9) cambiar el papel del paciente y la familia desde una postura pasiva a una postura activa (dar un significado al síntoma y construir una historia alternativa para que el paciente y la familia afronten el problema, puedan comunicarse mejor y disminuir tensiones en el ambiente familiar y recaídas en el paciente); 10) brindar herramientas de afrontamiento; 11) cierre y 12) seguimiento.

Después de la intervención se realizó un seguimiento telefónico bimestral. A los 12 meses fueron citados para evaluación clínica y aplicación del APGAR familiar. En este último mes se utilizaron pruebas de función pulmonar (espirometría) para determinar la persistencia de crisis asmáticas.

El tamaño de la muestra se calculó con el paquete estadístico Epidat 3.1, mediante la aplicación de tamaños de muestra para comparación de muestras emparejadas, considerando los siguientes valores: nivel de confianza del $95 \%$, poder del $80 \%$, una prevalencia de crisis asmáticas antes de la intervención familiar del $50 \%$ y una persistencia de las crisis después de la intervención del 10\%, obteniendo un tamaño de muestra de 23 pares.

La información fue tabulada en una hoja de Excel ${ }^{\circledR}$ y se exportó al paquete estadístico de STATA $10.0 \AA$ para el análisis estadístico de los datos y la generación de cuadros de salida. Se realizó un análisis univariado en la descripción de las características demográficas y clínicas de los niños con asma, y un análisis bivariado de los niños antes y después de la intervención utilizando las pruebas de Wilcoxon y McNemar. La significación estadística fue considerada con un valor de $p<$ 0,05 .

\section{RESULTADOS}

\section{Características socio-demográficas}

Los niños diagnosticados con asma y disfunción familiar (25) tenían en promedio 9,84 años (DS 1,34). El $64 \%$ provenían de familias de tipo nuclear y un $41,7 \%$ de estrato socioeconómico medio (tabla 1).

\section{Síntomas clínicos}

Se encontró una gran mejoría tras la intervención en la familia, con una disminución de los síntomas, con resultados estadísticamente significativos en todas las categorías (tabla 2).

\section{Espirometría}

Dos de las mediciones más importantes para la valoración del asma por medio de la espirometría, la Capacidad Vital Forzada (CVF) y el Volumen Espiratorio Forzado en el primer segundo (VEF1), mostraron un aumento significativo (tabla 3).

\section{Diagnóstico}

En la valoración en el primer momento por medio de la espirometría se encontró como resultado que el $68 \%$ de los niños tenían asma no controlada. 


\begin{tabular}{lcc}
\hline Variables & $\mathbf{n}$ & $\%$ \\
\hline Sexo & 15 & \\
Niños & 10 & 60,0 \\
Niñas & & 40,0 \\
Estrato socioeconómico & 1 & \\
Bajo & 6 & 4,0 \\
Bajo-medio & 10 & 24,0 \\
Medio & 4 & 40,0 \\
Medio-alto & 3 & 16,0 \\
Alto & 1 & 4,0 \\
No consta & & \\
Tipo de familia & 16 & 64,0 \\
Nuclear & 8 & 32,0 \\
No nuclear & 1 & 4,0 \\
Otra & 1 & \\
\hline
\end{tabular}

Tabla 1. Características sociodemográficasde los niños con asma incluidos en el programa de terapia familiar

\begin{tabular}{lccccc}
\hline \multicolumn{1}{c}{ Síntomas clínicos } & $\begin{array}{c}\text { Antes } \\
(\mathbf{\%})\end{array}$ & $\begin{array}{c}\text { Después } \\
(\%)\end{array}$ & $\begin{array}{c}\text { Diferencia de } \\
\text { proporciones }\end{array}$ & IC95\% & p \\
\hline Tos & 76,1 & 28,5 & 47,61 & $13-56$ & 0,0001 \\
Sibilancias & 71,4 & 9,5 & 61,90 & $25-71,1$ & 0,0000 \\
Dificultad respiratoria & 66,6 & 9,5 & 57,14 & $21,5-66,4$ & 0,0000 \\
Síntomas nocturnos & 61,9 & 19,0 & 42,85 & $25-71,1$ & 0,0000 \\
Utilización de beta-agonistas & 61,9 & 19,0 & 41,85 & $25-71,1$ & 0,0000 \\
\hline
\end{tabular}

Tabla 2. Síntomas clínicos de los niños con asma antes y después de la intervención familiar

\begin{tabular}{|c|c|c|c|c|c|}
\hline & \multicolumn{2}{|c|}{ Media (Desviación estándar) } & \multicolumn{2}{|c|}{ Mediana (Rango intercuartílico) } & \multirow[t]{2}{*}{$\mathbf{p}$} \\
\hline & Antes & Después & Antes & Después & \\
\hline CVF & $2,3(0,53)$ & $2,5(0,5)$ & $2,2(0,8)$ & $2,5(0,6)$ & 0,01 \\
\hline CVF\% & $0,98(0,21)$ & $0,97(0,18)$ & $0,96(0,22)$ & $0,95(0,28)$ & 0,82 \\
\hline VEF1 & $1,86(0,38)$ & $2,08(0,42)$ & $1,83(0,57)$ & $2,14(0,74)$ & 0,01 \\
\hline VEF1\% & $0,92(0,14)$ & $0,90(0,11)$ & $0,91(0,19)$ & $0,90(0,17)$ & 0,77 \\
\hline EI FEF & $216(47,4)$ & $247,5(45,9)$ & $217(93,0)$ & $245(79,5)$ & 0,00 \\
\hline FEF 25-75 & $1,88(0,74)$ & $2,15(0,86)$ & $1,72(1,24)$ & $2,14(1,64)$ & 0,57 \\
\hline FEF $25-75 \%$ & $0,81(0,30)$ & $0,75(0,25)$ & $0,77(0,46)$ & $0,77(0,37)$ & 0,02 \\
\hline MEF 25 & $3,33(0,78)$ & $3,79(0,87)$ & $3,14(1,35)$ & $3,60(1,46)$ & 0,01 \\
\hline MEF $25 \%$ & $0,85(0,17)$ & $0,85(0,15)$ & $0,86(0,27)$ & $0,86(0,20)$ & 0,97 \\
\hline MEF 50 & $2,39(0,97)$ & $2,55(0,86)$ & $2,17(1,73)$ & $2,66(1,22)$ & 0,88 \\
\hline MEF 50\% & $0,79(0,27)$ & $0,74(0,31)$ & $0,72(0,45)$ & $0,68(0,25)$ & 0,07 \\
\hline
\end{tabular}

Tabla 3. Resultados de la espirometría antes y después de la terapia familiar. CVF: Capacidad vital forzada. CVF \%: Porcentaje de la CVF. VEF1: Volumen espiratorio forzado en el primer segundo. VEF1 \%: Porcentaje del VEF1. FEF: Flujo espiratorio forzado. FEF 25-75: FEF entre el 25 y 75\% de la CVF. FEF 25-75 \%: Porcentaje del FEF 25-75. MEF 25: Flujo espiratorio medio al 25\% de la CVF. MEF 25\%: Porcentaje del MEF 25. MEF 50: Flujo espiratorio medio al $50 \%$ de la CVF. MEF $50 \%$ : Porcentaje del MEF 50.

\begin{tabular}{lccccc}
\hline \multicolumn{1}{c}{ Diagnóstico } & Antes (\%) & Después (\%) & $\begin{array}{c}\text { Diferencia de } \\
\text { proporciones }\end{array}$ & IC95\% & p \\
\hline Asma controlada & 32,0 & 95,2 & 47,61 & $13-56$ & 0,0001 \\
Asma no controlada & 68,0 & 4,0 & 61,90 & $25-71,1$ & 0,0000 \\
\hline
\end{tabular}

Tabla 4. Diagnóstico antes y después de la terapia familiar.

Después de la terapia familiar, al finalizar el año de seguimiento, se volvió a realizar la evaluación clínica con una persistencia del asma no controlada del $4 \%$, demostrando que la intervención familiar ayuda al control de la enfermedad en un gran porcentaje de los niños con asma (tabla 4).

\section{DISCUSIÓN}

El asma es una enfermedad con un gran componente orgánico pero a su vez es considerada como una enfermedad psicosomática. Tanto las características psicológicas de los niños afectados 
como las de sus cuidadores parecen contribuir al curso y a la aparición de la enfermedad ${ }^{18,19}$. Esta investigación encontró que la instauración de cambios en la dinámica familiar logra disminuir los síntomas y las crisis asmáticas y lleva a un mayor control de la enfermedad en los niños, es por esto que la evaluación de la dinámica familiar debe considerarse una parte fundamental en el abordaje integral del paciente pediátrico con asma ${ }^{5}$. En muchos casos no resulta suficiente el tratamiento exclusivo con medicamentos ${ }^{8,20} \mathrm{y}$, si se implementa un manejo interdisciplinario en el que se combinan el tratamiento farmacológico y la terapia familiar, se logra un mayor control de la enfermedad.

Con este estudio se buscaba evaluar a través de pruebas de función pulmonar la respuesta al tratamiento y la severidad del asma en niños antes y después de la intervención familiar. La espirometría fue la herramienta que se utilizó como método para la confirmación y el seguimiento de la enfermedad. Dos de las mediciones más importantes para la valoración del asma por medio de la espirometría, la CVF y el VEF1, mostraron un aumento significativo, demostrando que los niños presentaron una mejoría en su función pulmonar tras la terapia familiar al final del seguimiento.

Un estudio realizado en Suecia y otro en Inglaterra, que buscaban evaluar la eficacia de la terapia familiar como adyuvante a la terapia convencional en el asma, llegaron a la conclusión de que los niños que reciben terapia de familia, además de su medicación habitual, tienen mejor evolución y disminución en sus síntomas que los tratados sólo con la medicación ${ }^{18,21}$. En la primera fase de nuestro estudio se encontró que la disfunción familiar se presenta más frecuentemente en familias de niños con asma no controlada justificando la necesidad de incluir la intervención familiar dentro de la atención de estos pacientes ${ }^{22}$.

Adicionalmente varios estudios reportan que la implementación de la terapia familiar además del tratamiento médico ha mostrado que lleva a una mejoría de la función pulmonar en el volumen de flujo espiratorio máximo, las sibilancias durante el día, la evaluación clínica general y el número de días con deficiencia funcional'18,21,23.

De acuerdo con Minuchin ${ }^{24}$, la identificación de la relación entre las características de la organización familiar y las enfermedades psicosomáticas como el asma ha permitido diseñar y cambiar los patrones familiares que exacerban los síntomas, intervenir para cambiar el sistema de la familia y prevenir la recurrencia de éstos. En la presente investigación, las familias recibieron cuatro sesiones de terapia de familia con actividades y tareas con el fin de lograr un cambio en las relaciones familiares disfuncionales y en el curso y la evolución del asma, interviniendo los factores emocionales que empeoraban la enfermedad.

La instauración de un modelo de tratamiento que incluya tanto los medicamentos de elección para esta enfermedad pulmonar tan frecuente en los niños como la intervención de la dinámica familiar por medio de la terapia familiar llevará a una mejor evolución de los pacientes y un abordaje global de los síntomas y la aparición de crisis, al tener en cuenta los desencadenantes psicosociales que se pueden encontrar en una familia disfuncional.

Como limitaciones de este estudio hay que subrayar el hecho de que no se contó con un grupo control que permitiera evaluar si existían diferencias de acuerdo a recibir o no la intervención en terapia familiar. Por otra parte, no se controlaron los resultados de acuerdo al tipo de tratamiento farmacológico.

\section{BIBLIOGRAFÍA}

1. Reddel H, Barnes N, Barnes PJ, Bateman ED, Becker $A$, Bel E et al. Global Strategy for Asthma Management and Prevention, Global Initiative for Asthma (GINA) 2012. Disponible en: http://www.ginasthma.org/

2. British Thoracic Society. British guideline on the management of asthma; a national clinical guideline. London: BMJ Publ. Group. 2008.

3. Aranguren J, Callén M, Elorz J, Etxeberria A, Bautista J, Irizar I et al. Guía de Práctica Clínica sobre Asma. Disponible en: http://www.avpap.org/

4. Coffman K. Psychiatric issues in pulmonary disease. Psychiatr Clin North Am. 2002;25:89-127.

5. Guzmán-Pantoja JE, Barajas-Mendoza E, LuceGonzález EG, Valadez-Toscano FJ, GutiérrezRomán EA, Robles-Romero MA. Disfunción familiar en pacientes pediátricos con asma. Aten Primaria. 2008;40:543-8.

6. Schor EL; American Academy of Pediatrics Task Force on the Family. Family pediatrics: report of the Task Force on the Family. Pediatrics. 2003;111:154171.

7. Feldman JM, Ortega AN, Koinis-Mitchell D, Kuo AA, Canino G. Child and Family Psychiatric and Psychological Factors Associated With Child Physical Health Problems. J Nerv Ment Dis. 2010;198:272-9.

8. Rodríguez-Orozco AR, Kanán G, Vázquez ME, León A, Barbosa CE, Tejeda R. Perfil organizativo-funcional de la familia nuclear psicosomática con un hijo asmático. Salud Ment. 2008;31:63-8. 
9. Chen AY, Escarce JJ. Family structure and the treatment of childhood asthma. Med Care. 2008;46:174-84.

10. Clarke S-A, Calam R. The effectiveness of psychosocial interventions designed to improve health-related quality of life (HRQOL) amongst asthmatic children and their families: a systematic review. Qual Life Res. 2011;21:747-64.

11. Wirtberg I. Introduction to medical family therapy. Acta Paediatr Suppl. 2005; 94: 45-6.

12. Lask B, Kirk M. Childhood asthma: family therapy as an adjunct to routine management. J Fam Ther. 1979;1:33-49

13. Smilkstein G, Ashworth C, Montano D. Validity and reliability of the family APGAR as a test of family function. J Fam Pract. 1982;15:303-11.

14. Bellón JA, Delgado A, Luna JE, Lardelli P. Validez y fiabilidad del cuestionario de función familiar Apgarfamiliar. Aten Primaria. 1996;18:289-96.

15. Smilkstein G. The family APGAR: a proposal for a family function test and its use by physicians. J Fam Pract. 1978;6:1231-9.

16. Sánchez y Gutiérrez, D. Terapia familiar: modelos y técnicas. México, D.F.: Manual Moderno. 2000.

17. Sluzki CE. Transformations: a blueprint for narrative changes in therapy. Fam Process. 1992;31:217-30.
18. Lask B, Matthew D. Childhood asthma. A controlled trial of family psychotherapy. Arch Dis Child. 1979;54:116-9.

19. Tibosch MM, Verhaak CM, Merkus PJFM. Psychological characteristics associated with the onset and course of asthma in children and adolescents: A systematic review of longitudinal effects. Patient Educ Couns. 2011;82:11-9.

20. Ng SM, Li AM, Lou VWQ, Tso IF, Wan PYP, Chan DFY. Incorporating family therapy into asthma group intervention: a randomized waitlist-controlled trial. Fam Process. 2008;47:115-30.

21. Gustafsson PA, Kjellman NI, Cederblad M. Family therapy in the treatment of severe childhood asthma. J Psychosom Res. 1986;30:369-74.

22. Berbesí DY, García MM, Segura AM, Posada R. Evaluación de la dinámica familiar en familias de niños con diagnóstico de asma. Rev Col Psiqui. 2013;42:63-71.

23. Yorke J, Shuldham C. Family therapy for asthma in children. Cochrane Database Syst Rev. 2005;2:CD000089.

24. Liebman R, Minuchin S, Baker L. The use of structural family therapy in the treatment of intractable asthma. Am J Psychiatry. 1974;131:535-40. 